\title{
A Randomized, Double-Blind, Placebo-Controlled, First-in-Human Clinical Trial to Assess Safety, Tolerability, and Pharmacokinetics of LY-CovMab, a Potent Human Neutralizing Antibody Against SARS- CoV-2
}

Qian Zhang $\cdot$ Renpeng Zhou $\cdot$ Jingjing Yang $\cdot$ Changlin Dou $\cdot$ Tianyi Gan $\cdot$ Fujia Liu $\cdot$ Baihui Hu $\cdot$ Deyong Song $\cdot$ Chao $\mathrm{Lu} \cdot$ Wei Hu (1)

Received: November 1, 2021 / Accepted: November 23, 2021 / Published online: December 8, 2021

(C) The Author(s) 2021

\section{ABSTRACT}

Introduction: We aimed to evaluate the safety, tolerability, pharmacokinetics, and immunogenicity of a single dose of LY-CovMab in Chinese healthy adults.

Methods: We conducted a phase 1, randomized, dose-escalation, placebo-controlled trial in 42 volunteers, $18-45$ years of age, and 40 out of 42 received a single dose of LY-CovMab or placebo with LY-CovMab at a dose of $30 \mathrm{mg}$,

Qian Zhang and Renpeng Zhou contributed equally to this work and should be considered co-first authors.

Supplementary Information The online version contains supplementary material available at https:// doi.org/10.1007/s40121-021-00572-x.

Q. Zhang $\cdot$ R. Zhou $\cdot$ J. Yang $\cdot$ W. Hu ( $\bowtie)$ Department of Clinical Pharmacology, The Second Hospital of Anhui Medical University, Hefei, People's Republic of China e-mail: huwei@ahmu.edu.cn

Q. Zhang · R. Zhou · J. Yang · W. Hu

Anhui Provincial Institute of Translational

Medicine, Hefei, People's Republic of China

C. $\mathrm{Lu}(\bowtie)$

First Affiliated Hospital, Anhui University of Science and Technology, Hefei, People's Republic of China e-mail: luchao@aust.edu.cn

C. Dou - T. Gan · F. Liu - B. Hu · D. Song Shandong Boan Biotechnology Co., Ltd., Yantai, People's Republic of China
$150 \mathrm{mg}, 600 \mathrm{mg}, 1200 \mathrm{mg}$, and $2400 \mathrm{mg}$. There were ten subjects in each group receiving LYCovMab or placebo in a $4: 1$ ratio with the exception that the $30 \mathrm{mg}$ group had two subjects both receiving LY-CovMab.

Results: Among the 42 randomized participants, 40 received an injection with 32 administered LY-CovMab and 8 administered placebo. A total of 18 drug-related treatment-emergent adverse events (TEAEs) were reported in 12 subjects $(30.0 \%)$, including protein urine present $(25 \%, 10 / 40)$ and blood creatinine increased $(7.5 \%, 3 / 40)$. The incidence of drugrelated TEAE in each dosage group was as follows: $150 \mathrm{mg}(28.6 \%, 2 / 7), 600 \mathrm{mg}(25 \%, 2 / 8)$, $1200 \mathrm{mg}(14.3 \%, 1 / 7), 2400 \mathrm{mg}(50 \%, 4 / 8)$, and placebo $(37.5 \%, 3 / 8)$. All drug-related TEAEs were grade 1 , and most of them were recovering/resolving or recovered/resolved without taking action. The serum exposure of LY-CovMab ( $\left.\mathrm{C}_{\max }, \mathrm{AUC}_{0-\text { last}}, \mathrm{AUC}_{0 \text {-inf }}\right)$ after intravenous infusion increased in an approximately proportional manner as the dose increased from 150 to $2400 \mathrm{mg}$. The elimination half-life $\left(t_{1 / 2}\right)$ value did not differ among different dose cohorts and was estimated to be around 28.5 days.

Conclusions: A single dose of LY-CovMab was shown to be safe and well tolerated in Chinese healthy adults. The pharmacokinetic (PK) profiles of LY-CovMab in healthy adults showed typical monoclonal antibody distribution and 
elimination characteristics. LY-CovMab demonstrated dose proportionality.

Trial Registration: ClinicalTrial.gov Identifier NCT04973735.

Keywords: SARS-CoV-2; Monoclonal neutralizing antibody; COVID-19; LY-CovMab; Safety; Pharmacokinetics; Immunogenicity

\section{Key Summary Points}

\section{Why carry out this study?}

LY-CovMab is a fully humanized monoclonal antibody manufactured by recombinant technology and used to counteract COVID-19. Preclinical data from LY-CovMab showed it could effectively inhibit pseudovirus and authentic virus infection in vitro and in vivo, which suggested that it could be a promising SARS-CoV-2 neutralizing antibody candidate.

This study evaluated the safety, tolerability, pharmacokinetics, and immunogenicity of a single dose of LYCovMab in Chinese healthy adults.

\section{What was learned from the study?}

Results of this study showed that a single dose of LY-CovMab was safe and well tolerated in Chinese healthy adults. The pharmacokinetic (PK) profiles of LYCovMab in healthy adults showed typical monoclonal antibody distribution and elimination characteristics. LY-CovMab demonstrated dose proportionality. Clinically, LY-CovMab could be a promising SARS-CoV-2 neutralizing antibody candidate against SARS-CoV-2.

This first-in-human clinical study disclosed the specific PK profile of LYCovMab in Chinese healthy adults, which combined with the safe and well-tolerated characteristics support that LY-CovMab could be of clinical potential building on its strong preclinical evidence of inhibiting SARS-CoV-2 infection.

\section{INTRODUCTION}

As the pathogen of novel coronavirus disease (COVID-19), severe acute respiration syndrome coronavirus 2 (SARS-CoV-2) is continuing to spread and cause the global pandemic with more than 200 million confirmed COVID-19 cases including above 4.0 million deaths so far [1]. However, we are still facing few options for effective medications.

Remdesivir, an antiviral agent, is currently the only therapeutic drug that is approved by the US Food and Drug Administration (FDA) for the treatment of COVID-19 [2]. Dexamethasone, a corticosteroid, has been strongly recommended for use in hospitalized patients who require supplemental oxygen by the FDA and World Health Organization (WHO) [3]. Furthermore, the FDA has issued Emergency Use Authorizations (EUAs) [4] for the SARS-CoV-2 neutralizing antibody, including REGEN-COV for both treatment of patients with mild-tomoderate COVID-19 and post-exposure prophylaxis of COVID-19 in individuals who are at high risk to progression to severe COVID-19 and sotrovimab for the treatment of patients with mild-to-moderate COVID-19. The FDA has also approved the first COVID-19 vaccine known as Pfizer-BioNTech COVID-19 vaccine and will now be marketed as Comirnaty and issued EUAs for two vaccines, including mRNA-1273 (Moderna) and Janssen COVID-19 Vaccine (Janssen Biotech, Inc.) for COVID-19 prevention. Although remdesivir, SARS-CoV-2 neutralizing antibody, and vaccines have offered both therapeutic and preventive benefits, a large number of people are still suffering the terrible pandemic, which causes an ever-surging number of patients with COVID-19, creating a large unmet medical need.

LY-CovMab, developed by Shandong BoAn Biotechnology Co., Ltd., is a fully humanized monoclonal antibody manufactured by recombinant technology and used to counteract COVID-19 [5]. It belongs to the IgG4 subtype and consists of two heavy chains and two light chains with a molecular weight of approximately $147 \mathrm{kDa}$. Given that antibody-dependent enhancement (ADE) is a potential risk for 
antibody therapeutics against SARS-CoV-2 infection, FALA mutation was introduced to potentially abrogate the ADE effect in the LYCovMab. Structural analysis revealed that LYCovMab recognizes an epitope overlapping with angiotensin converting enzyme 2 (ACE2)binding sites in SARS-CoV-2 receptor-binding domain (RBD) in the spike protein. LY-CovMab blocks the interaction by binding all three RBDs of one SARS-CoV-2 spike trimer simultaneously [5]. In preclinical study, a single-dose administration of LY-CovMab dramatically reduced RNA viral loads of $B A L B / c$ mice who received MAScp6 challenge and resulted in a 34,914-fold and 693-fold reduction of viral titers in the lungs and tracheas at 3 days post infection compared with a single-dose administration of phosphate-buffered saline [5]. Furthermore, LYCovMab has demonstrated a good pharmacokinetic profile following a single-dose intravascular injection in mice and rhesus monkeys [5]. The purpose of this study is to assess safety, tolerability, and pharmacokinetics of LY-CovMab in Chinese healthy adults.

\section{METHODS}

\section{Study Design and Participants}

This phase 1, randomized, double-blind, single ascending dose, placebo-controlled study included 42 randomized Chinese healthy adults aged 18-45 years old from the Second Hospital of Anhui Medical University of China. The study was conducted in accordance with the principles of Good Clinical Practice and the Declaration of Helsinki of 1964 and its later amendments. The Ethics Committee of the Second Hospital of Anhui Medical University approved the trial design, and eligible participants provided written informed consent before participating (reference numbers SL-YW2020113).

\section{Randomization and Masking}

The phase 1, dose-escalation study included five dose groups (30 mg, $150 \mathrm{mg}, 600 \mathrm{mg}, 1200 \mathrm{mg}$, and $2400 \mathrm{mg}$ ) with ten eligible adults in each except the $30 \mathrm{mg}$ group which had two subjects both received the investigational drug. The subjects in the $150 \mathrm{mg}$ group were started to be enrolled if $30 \mathrm{mg}$ group subjects were both confirmed to be safe by investigators on day 8 after administration. In each of the other four groups (i.e., $150 \mathrm{mg}, 600 \mathrm{mg}, 1200 \mathrm{mg}$, and $2400 \mathrm{mg}$ ), the enrollment details were as follows. Two out of ten healthy adults were enrolled first as sentinels in the $150 \mathrm{mg}$ group, who were randomized to receive the investigational drug or placebo. After the investigator confirmed the acceptable safety and tolerability on day 4 post dose, the remaining eight subjects were then randomly enrolled to receive LYCovMab or placebo in a $7: 1$ ratio. The two sentinels of the $600 \mathrm{mg}$ group could only be enrolled when the acceptable safety of eight subjects in the $150 \mathrm{mg}$ group was confirmed by the investigator on day 8 after administration. The enrollment order of the other three groups (600 mg, $1200 \mathrm{mg}$, and $2400 \mathrm{mg}$ ) was the same as that of the $150 \mathrm{mg}$ group. Participants were hospitalized until day 8 after administration and came back for follow-up visit at day 15 (D15), D22, D29, D36, D43, D50, D57, D71, and D99 for $150 \mathrm{mg}, 600 \mathrm{mg}, 1200 \mathrm{mg}$, and $2400 \mathrm{mg}$ groups, whereas follow-up visits of the $30 \mathrm{mg}$ group were at D15, D29, D43, D50, D57, and D71. During the study, blood samples were collected for pharmacokinetic and immunogenic analyses, and participants' vital signs, physical examination, 12-lead electrocardiogram (ECG), laboratory tests, and adverse events were recorded.

The Interactive Web Response System (IWRS) was used for randomization. An unblinded statistician prepared the randomization assignment table by block randomization method using Statistical Analysis System (SAS) version 9.4 and uploaded the randomization assignment table to IWRS. The investigator participating in the trial logged in IWRS after each eligible subject was screened to obtain the corresponding randomization number and drug number of the trial.

The trial adopted a double-blinded design, and the investigational drug and placebo were processed in a blinded way. An unblinded 
statistician or other relevant trained and authorized professionals conducted blindness work of drugs according to the drug randomization plan and randomization table. The whole blindness process followed strictly the standard operating procedure (SOP) for drug blindness, and the whole blindness process was recorded. This study was a phase 1 clinical trial and an inferential statistical test was not performed. There was no formal hypothesis for the sample size.

\section{Main Entry Criteria}

Eligible participants were male and female Chinese healthy adults aged 18-45 years old with weight of male participants at least $50 \mathrm{~kg}$, weight of female participants at least $45 \mathrm{~kg}$, and body mass index (BMI) $19.0 \leq \mathrm{BMI} \leq 26.0 \mathrm{~kg} / \mathrm{m}^{2}$. There was no abnormality, or no clinically significant abnormality in vital signs, physical examination, laboratory tests, and 12-lead ECG in participants during the screening period. All sexually active patients in the study were required to use medically acceptable contraception.

Participants were excluded if they had any of the follows: any surgery within 8 weeks prior to screening or any surgery planned during the trial, and the investigator believed that such surgery could bring potential risk to the subjects; use of any prescription drug within 28 days and any over-the-counter drug, including health care products within 7 days prior to administration of the investigational drug; prior history or current evidence of serious disease or chronic disease; having COVID-19 or recovered from it or being positive for SARS-CoV-2 nucleic acid or serum SARS-CoV-2-specific antibodies; known history of allergies to any ingredient of the investigational drug, and so on. Detailed entry criteria are listed in the protocol in the Supplementary Material.

\section{Assessment of Safety}

The primary objectives were evaluation of safety and tolerability of a single dose of LY-CovMab in healthy subjects. The safety evaluations performed at each visit included vital signs, physical examination, laboratory tests (hematology, blood chemistry, routine urine test, and coagulation tests), 12-lead ECG, and adverse events. Adverse events (AE) were coded using Medical Dictionary for Regulatory Activities (MedDRA). The severity of $\mathrm{AE}$ was graded according to Common Terminology Criteria for Adverse Events (CTCAE 5.0). The secondary objectives were evaluation of PK characteristics and immunogenicity of a single dose of LYCovMab in healthy subjects.

\section{Assessment of PK}

Blood samples were collected before administration, $0.5 \mathrm{~h}$ (D1) after administration, immediately after the end of administration (D1), $4 \mathrm{~h}$ (D1), $8 \mathrm{~h}$ (D1), $24 \mathrm{~h}$ (D2), $48 \mathrm{~h}$ (D3), $72 \mathrm{~h}$ (D4), $168 \mathrm{~h}$ (D8), $336 \mathrm{~h}$ (D15), $504 \mathrm{~h}$ (D22), $672 \mathrm{~h}$ (D29), 840 h (D36), 1008 h (D43), 1176 h (D50), $1344 \mathrm{~h}$ (D57), $1680 \mathrm{~h}$ (D71), and $2352 \mathrm{~h}$ (D99) after administration. A 2-mL sample of whole blood of was collected at each time point, and the concentration of LY-CovMab in the serum was measured.

The serum concentrations of LY-CovMab were measured by an enzyme-linked immunosorbent assay (ELISA) at Labcorp Pharmaceutical Research and Development (Shanghai) Co., Ltd. (Shanghai, China). The lower limit of quantification (LLOQ) was $200 \mathrm{ng} / \mathrm{mL}$. The quantitative linear range and the calibration curve assay range were from 200 to $8000 \mathrm{ng} / \mathrm{mL}$. For the PK analysis, concentrations less than the LLOQ were set to zero. All measurements were accurate to the measure of $-10.9 \%$ to $24 \%$. The coefficients of variation for intra- and inter-day precision were $2.0-10.6 \%$ and $6.2-12 \%$, respectively.

Then the drug concentration data were analyzed to obtain the PK parameters by a noncompartmental analysis method using Phoenix WinNonlin software V8.1 (Pharsight Corp., Mountain View, CA, USA). The actual sampling time was used for the calculation of PK parameters, including maximum concentration $\left(C_{\text {max }}\right)$, time to reach $C_{\max }\left(T_{\max }\right)$, area under the serum concentration-time curve from time 
zero to the time of the last quantifiable concentration $\left(\mathrm{AUC}_{0-\text { last }}\right)$, the elimination rate constant $\left(\lambda_{z}\right)$, area under the serum concentration-time curve from time zero to infinity $\left(\mathrm{AUC}_{0-\text { inf }}\right)$, elimination half-life $\left(t_{1 / 2}\right)$, clearance $(\mathrm{CL})$, volume of distribution $\left(V_{\mathrm{z}}\right)$, and mean residence time (MRT), etc.

\section{Assessment of Immunogenicity}

Blood samples were collected from all subjects before administration and at $336 \mathrm{~h}$ (D15), $672 \mathrm{~h}$ (D29), $1008 \mathrm{~h}$ (D43), $1344 \mathrm{~h}$ (D57), and $1680 \mathrm{~h}$ (D71) after administration. Blood was also collected from subjects in the $150 \mathrm{mg}, 600 \mathrm{mg}$, $1200 \mathrm{mg}$, and $2400 \mathrm{mg}$ groups on D99 post dose. A 4-mL sample of whole blood was collected at each time point to detect anti-drug antibodies (ADA). The serum concentrations of LY-CovMab were determined by a validated ELISA. Samples with ADA positivity were further tested for the presence or absence of neutralizing antibodies using validated assays. The titer of positive ADA samples was also evaluated.

\section{Statistical Analysis}

Statistical analysis was conducted by SAS v9.4 for Windows. The safety analysis was mainly summarized by descriptive statistics. The number of subjects, onset time, and incidence of positive ADA and the corresponding titer of ADA, were described by treatment groups at scheduled sampling time points. Descriptive statistics of PK parameters were summarized by dose groups. An exponential power model and/ or dose normalization method was used to primarily evaluate the main pharmacokinetic parameter (AUC and $C_{\max }$ ).

\section{RESULTS}

\section{Subject Disposition}

A total of 255 Chinese healthy volunteers signed the informed consent form with 213 failures upon screening. A total of 42 participants were randomly assigned to the groups; two subjects dropped out before administration. The dosing details were as follows: two subjects received $30 \mathrm{mg}$ LY-CovMab, seven subjects received $150 \mathrm{mg}$ LY-CovMab, eight subjects received $600 \mathrm{mg}$ LY-CovMab, seven subjects received $1200 \mathrm{mg}$ LY-CovMab, eight subjects received $2400 \mathrm{mg}$ LY-CovMab, and eight subjects received placebo. In total, 37 out of 42 participants completed the study and 5 participants withdrew from the study (Fig. 1).

There were 30 men $(71.4 \%)$ and 12 women (28.6\%) among the 42 randomized participants with the average age (mean \pm SD) of $27.5 \pm 6.94$ years. Among 40 adults who received an injection, the average height (mean $\pm \mathrm{SD}$ ), weight (mean $\pm \mathrm{SD}$ ), and BMI $($ mean \pm SD) $\quad$ were $\quad 171.03 \pm 8.47 \mathrm{~cm}$, $65.00 \pm 8.93 \mathrm{~kg}$, and $22.16 \pm 1.93 \mathrm{~kg} / \mathrm{m}^{2}$ respectively. The detailed demographics and baseline characteristics are shown in Table 1.

\section{PK Results}

As shown in Fig. 2, the mean serum concentrations of LY-CovMab in the healthy volunteers increased as the dose increased. The PK parameters of LY-CovMab are summarized descriptively by dose in Table 2 . Following a single intravenous infusion in the $150 \mathrm{mg}$ group $(N=7), 600 \mathrm{mg}$ group $(N=8), 1200 \mathrm{mg}$ group $(N=7)$, and $2400 \mathrm{mg}$ group $(N=8), C_{\max }$ $($ mean $\pm \mathrm{SD})$ were values $52.19 \pm 11.91$, $245.00 \pm 63.43,430.14 \pm 51.41$, and $936.88 \pm$ $112.81 \mu \mathrm{g} / \mathrm{mL}$, respectively; $\mathrm{AUC}_{0-\text { last }}$ (mean \pm SD) values were $27,418.12 \pm 4082.81$, $122,238.81 \pm 11,546.69, \quad 226,136.19 \pm$ $59,051.21$, and $480,108.56 \pm 55,534.41 \mathrm{~h} \cdot \mu \mathrm{g} /$ $\mathrm{mL}$, respectively; and $\mathrm{AUC}_{0-\text { inf }}($ mean $\pm \mathrm{SD}$ ) values were $31,448.24 \pm 4445.35,130,964.19$ $\pm 11,764.57, \quad 271,426.16 \pm 53,670.62$, and $545,192.05 \pm 79,253.12 \mathrm{~h} \cdot \mu \mathrm{g} / \mathrm{mL}$, respectively. The mean half-life $\left(t_{1 / 2}\right)$ was around 28.5 days and ranged from 24.4 days $(586 \mathrm{~h})$ to 34.2 $(821 \mathrm{~h}$ ) days across dose groups (Table 2 ).

A power model method was used to assess the dose proportionality based on the log transformed $C_{\max }, \mathrm{AUC}_{0-\text { last}}$, and $\mathrm{AUC}_{0-\text { inf }}$. With the dose ranging from 150 to $2400 \mathrm{mg}$, the point estimates of $C_{\max }, \mathrm{AUC}_{0-\text { last}}$, and 


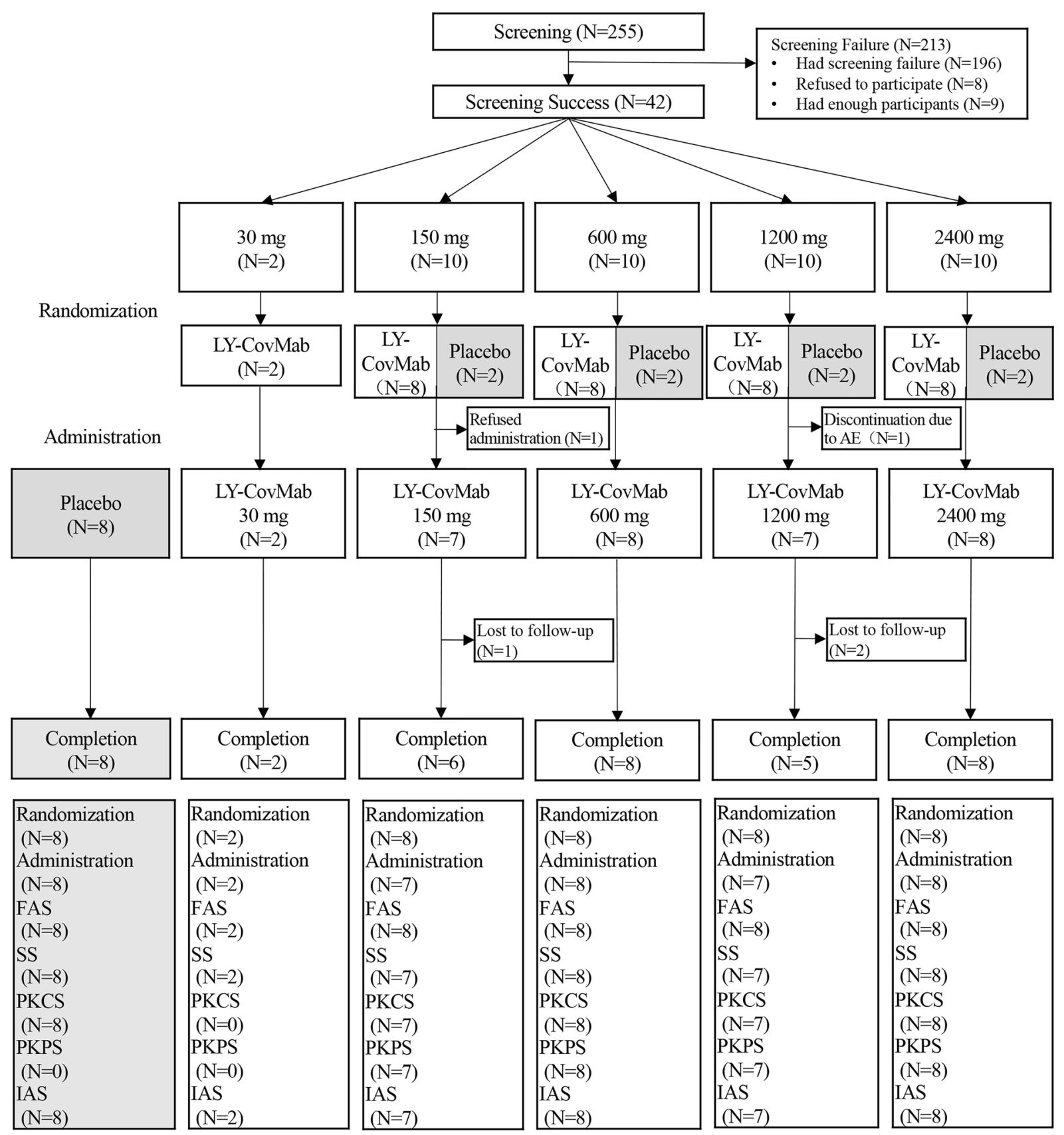

Fig. 1 Study design and flow diagram. Note: (1) Full analysis set (FAS) was defined as subjects who were randomized. (2) Safety set (SS) was defined as subjects who were randomized and received at least one dose of study drug. (3) Pharmacokinetics concentration set (PKCS) was defined as subjects who received at least one dose of study drug and had at least one evaluable serum concentration. (4) Pharmacokinetics parameter set (PKPS) was defined as

subjects who received at least one dose of study drug and had sufficient serum concentration data to obtain at least one PK parameter and had no major protocol deviations that affected the evaluation of PK parameters. (5) Immunogenicity analysis set (IAS) was defined as subjects who received at least one dose of study drug and had at least one evaluable data point of immunogenicity after administration 


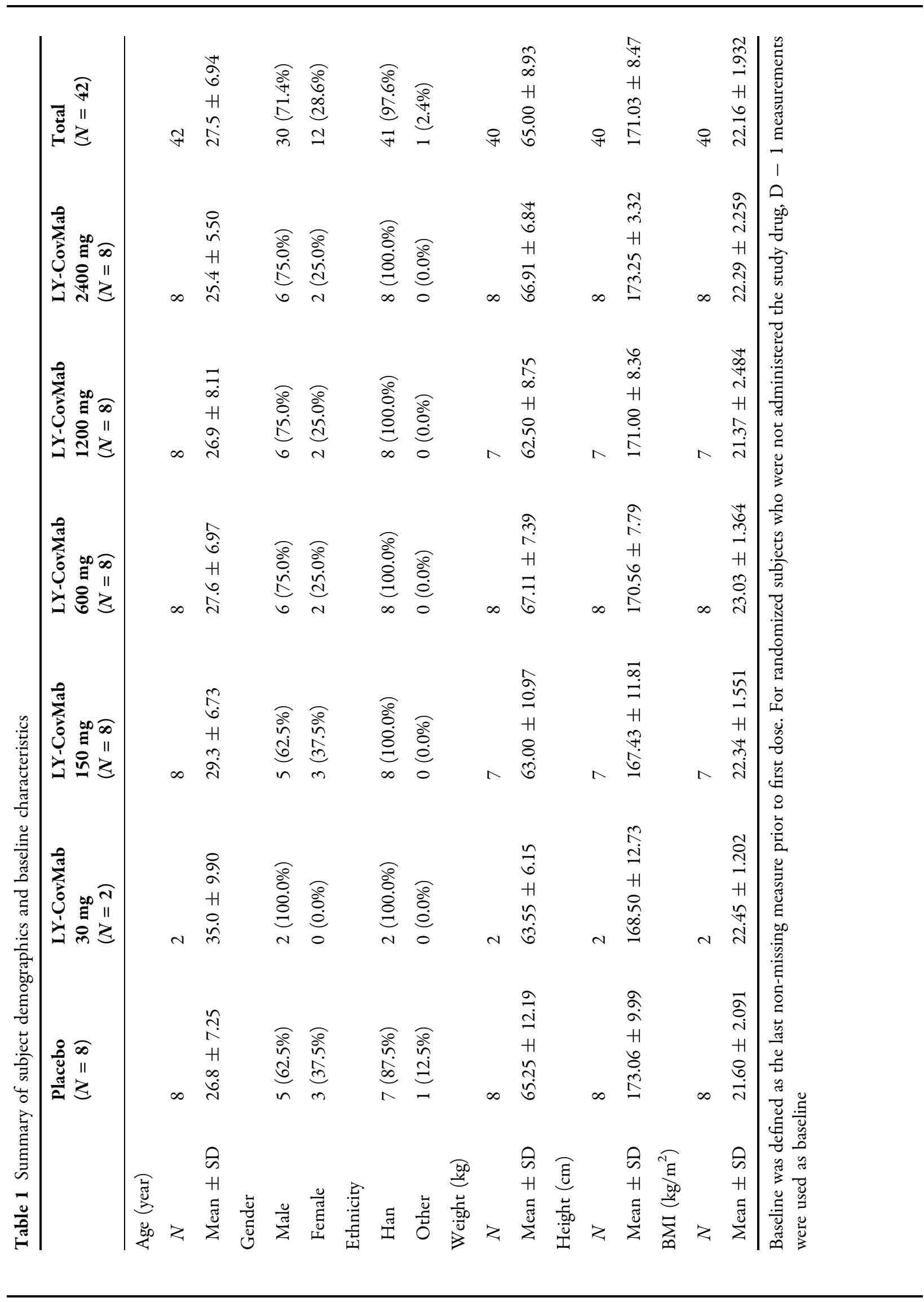



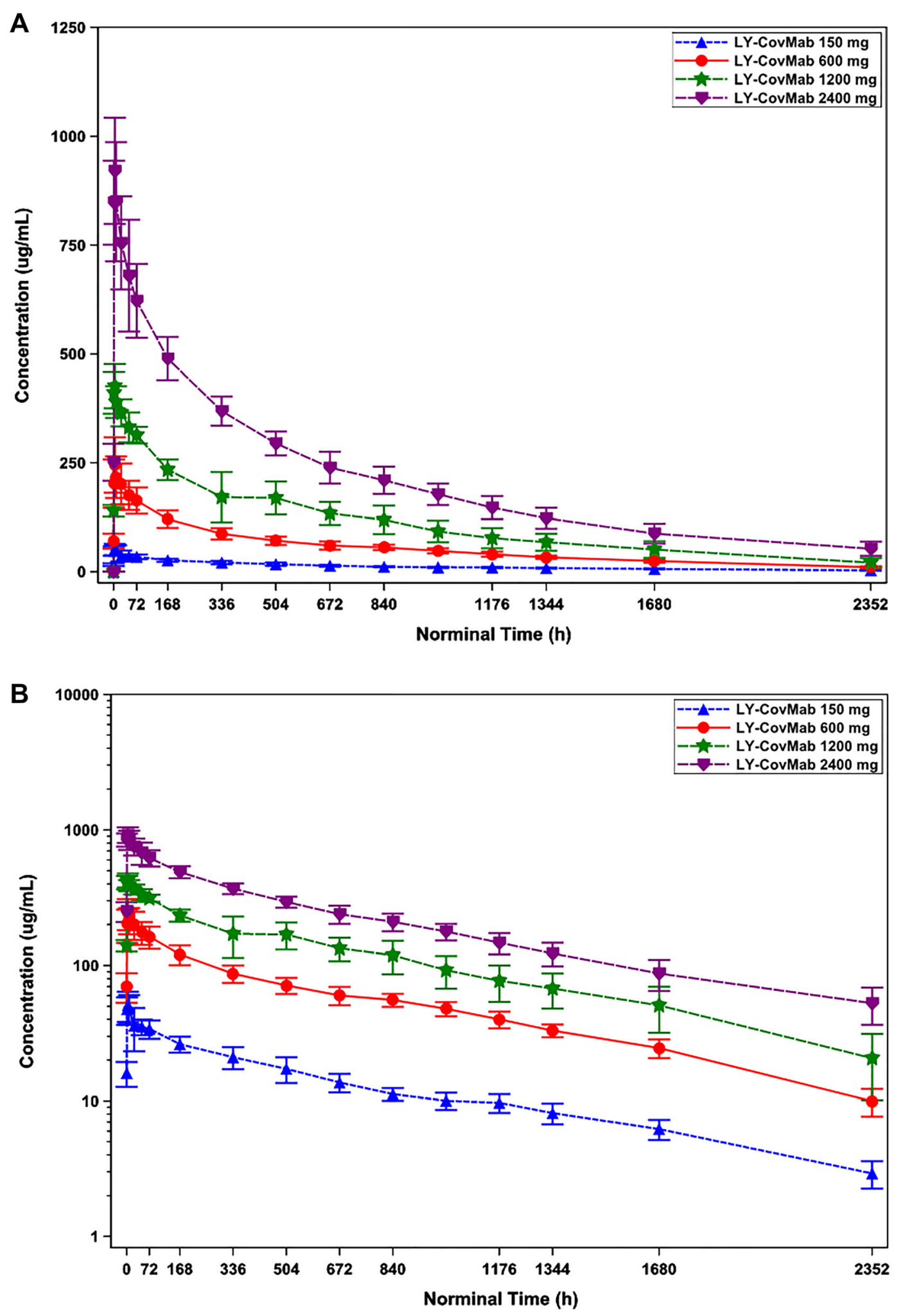
४Fig.2 Mean ( \pm standard deviation $[S D]$ ) of LY-CovMab serum concentration-time profiles in healthy participants following single-dose intravenous infusions at $150 \mathrm{mg}$, $600 \mathrm{mg}, 1200 \mathrm{mg}$, and $2400 \mathrm{mg}$ : a linear scale and b semilog scale

$\mathrm{AUC}_{0-\text { inf }}$ were $1.04,1.02$, and 1.03 , respectively. The $95 \%$ confidence interval (CI) of the point estimates contains 1 . Therefore, exposure parameters $\left(C_{\max }\right.$ and AUC) were considered to increase in an approximately proportional manner as the dose increased (Table 3 ).

\section{Immunogenicity Results}

ADA tested positive in $12.5 \%$ of the participants $(5 / 40)$, including one subject in the $150 \mathrm{mg}$ group, two subjects in $600 \mathrm{mg}$ group, one subject in the $1200 \mathrm{mg}$ group and one subject in the $2400 \mathrm{mg}$ group. One subject in the $1200 \mathrm{mg}$ group was tested ADA positive before dosing and was not considered to be caused by LYCovMab. The ADA positivity did not impact on the serum concentration or safety profile of LYCovMab (Table 4). This will be confirmed with neutralizing antibody analysis in future studies.

\section{Safety Results}

A total of 40 participants received an injection and were analyzed in the safety set (SS) with 32 in the investigational drug group and 8 in the placebo group. Thirty-one (77.5\%) participants reported 99 TEAEs, including 5 events in 2 (100.0\%) participants in the $30 \mathrm{mg}$ group, 13 events in $7(100.0 \%)$ participants in the $150 \mathrm{mg}$ group, 22 events in $5(62.5 \%)$ participants in the $600 \mathrm{mg}$ group, 3 events in $2(28.6 \%)$ participants in the $1200 \mathrm{mg}$ group, 33 events in 7 $(87.5 \%)$ participants in the $2400 \mathrm{mg}$ group, and 23 events in $8(100 \%)$ participants in the placebo group (Table 5). All the TEAEs have been reviewed and approved by the local ethics committees.

TEAEs sorted by descending incidence in each system organ class (SOC) included investigations, infections and infestations, cardiac disorders, gastrointestinal disorders, and musculoskeletal and connective tissue disorders. The most common three TEAEs in LY-CovMab groups were protein urine present, blood triglycerides increased, and white blood cells urine positive. The incidence of protein urine present was higher in the $2400 \mathrm{mg}$ group (Table 5).

Among TEAEs, 90 events in 29 participants were classified as grade 1 and 9 events in 5 participants were classified as grade 2 . The grade 2 TEAEs included four events in one participant in the $150 \mathrm{mg}$ group, one event in one participant in the $1200 \mathrm{mg}$ group, and four events in three participants in placebo group. Most TEAEs were recovered/resolved without taking action. There were no deaths, withdrawals due to TEAEs, and no serious adverse events (SAE), serious drug-related TEAEs, or other significant adverse events occurred during the study (Table 6).

Twelve (30.0\%) participants out of 40 experienced 18 drug-related TEAEs. There were two events in 2 (28.6\%) participants in the $150 \mathrm{mg}$ group, six events in $2(25.0 \%)$ participants in the $600 \mathrm{mg}$ group, one event in 1 (14.3\%) participant in the $1200 \mathrm{mg}$ group, five events in 4 (50.0\%) participants in the $2400 \mathrm{mg}$ group, and four events in $3(37.5 \%)$ participants in the placebo group (Table 7).

\section{DISCUSSION}

Preclinically reported data from LY-CovMab effectively inhibiting pseudovirus and authentic virus infection in vitro and in vivo by interfering with the mechanism that the virus attaches to the host cell suggested that it could be a promising SARS-CoV-2 neutralizing antibody candidate [5]. Consistent with our preclinical finding, LY-CovMab displayed a good safety and pharmacokinetic profile in the first-in-human study, which supported its clinical potential.

In this study, the safety of a single-dose injection of LY-CovMab was as expected for a full-length recombinant human antibody in all five dose groups from 30 to $2400 \mathrm{mg}$ [5]. We did not observe injection site reactions and hypersensitivity reactions, which have been reported 


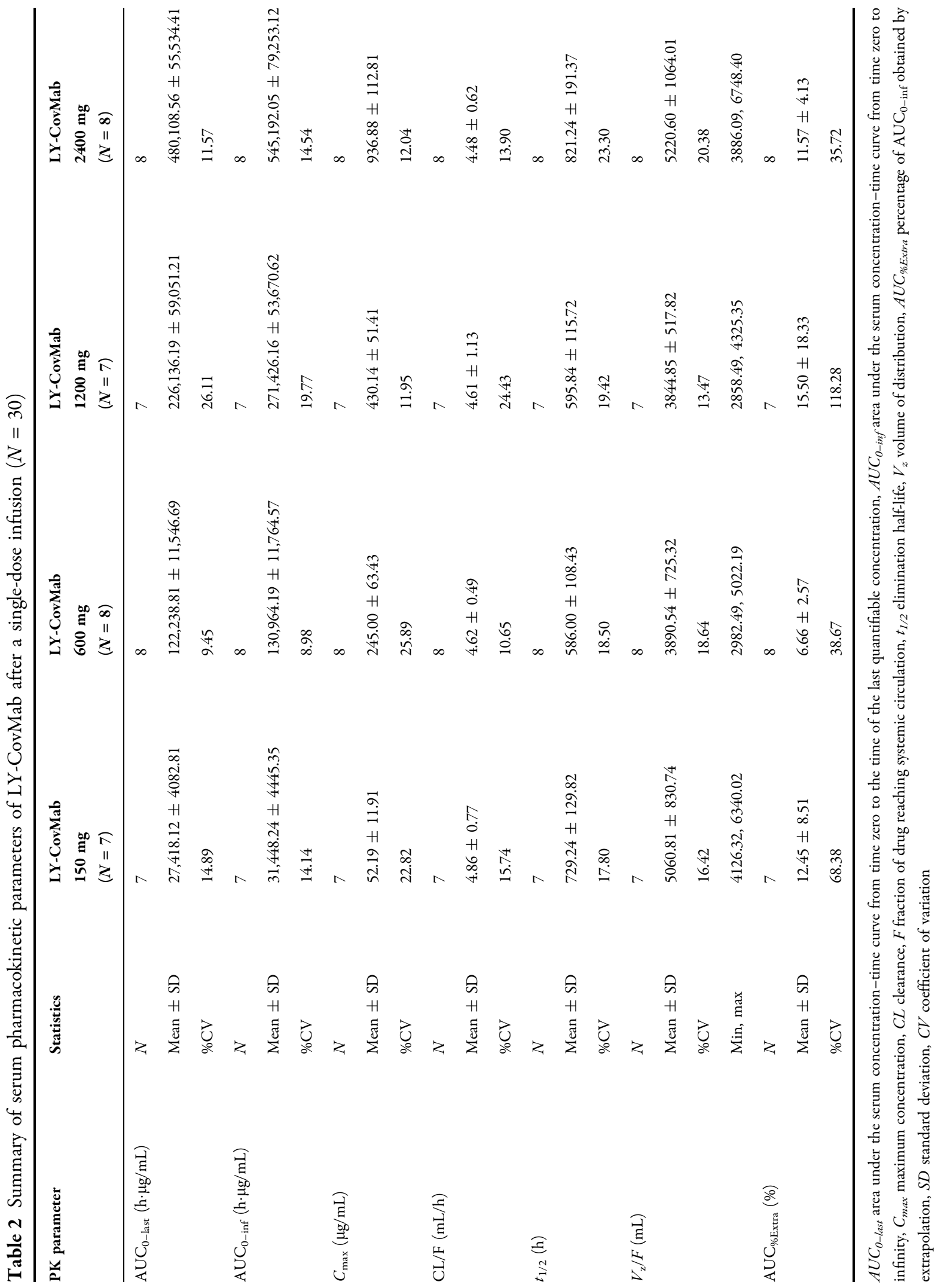


Table 3 Analysis of dose proportionality: model parameters $(N=40)$

\begin{tabular}{lcccc}
\hline $\begin{array}{l}\text { PK } \\
\text { parameter }\end{array}$ & Intercept & Slope & 95\% CI & 90\% CI \\
\hline $\begin{array}{l}C_{\max }(\mu \mathrm{g} / \\
\mathrm{mL})\end{array}$ & -1.24 & 1.04 & $0.97,1.11$ & $0.98,1.10$ \\
$\begin{array}{c}\mathrm{AUC} \mathrm{C}_{0-\mathrm{last}} \\
(\mathrm{h} \cdot \mu \mathrm{g} / \mathrm{mL})\end{array}$ & 5.10 & 1.02 & $0.96,1.09$ & $0.97,1.08$ \\
$\begin{array}{c}\mathrm{AUC} \mathrm{C}_{0-\mathrm{inf}} \\
(\mathrm{h} \cdot \mu \mathrm{g} / \mathrm{mL})\end{array}$ & 5.19 & 1.03 & $0.97,1.08$ & $0.98,1.08$ \\
\hline
\end{tabular}

Subjects in the placebo group were not analyzed for PK pharmacokinetic parameters

$A U C_{0 \text {-last }}$ area under the serum concentration-time curve from time zero to the time of the last quantifiable concentration, $A U C_{0-\text { inf }}$ area under the serum concentration-time curve from time zero to infinity, $C_{\max }$ maximum concentration, $C I$ confidential interval

in studies of other neutralizing antibodies $[6,7]$. Infusion-related hypersensitivity reactions were reported in nine patients, of whom six were in the bamlanivimab monotherapy groups, two in the bamlanivimab and etesevimab group, and one in the placebo group [6]. The study of Regeneron's REGN-COV2, a neutralizing antibody cocktail against SARS-CoV-2, also showed grade 2 or higher infusion-related reactions and hypersensitivity reactions in both REGN-COV2 dose groups and the placebo group [7]. There were no deaths, SAEs, and discontinuation due to TEAEs through our whole study. All drug-related TEAEs were grade 1 , and most of them were recovering/resolving or recovered/resolved without taking action. The incidence of drugrelated TEAEs in the $2400 \mathrm{mg}$ group $(50 \%, 4 / 8)$ and placebo group $(37.5 \%, 3 / 8)$ was similar whereas the incidence of drug-related TEAEs in the other LY-CovMab groups was lower than that of placebo group (Table 7). The severity of drug-related TEAEs was roughly balanced between LY-CovMab dose groups and placebo group (Table 7).

Drug-related TEAEs, including protein urine present (10 participants, 15 events) and blood creatinine increased (3 participants, 3 events), occurred in this study and all of the events were classified as CTCAE grade 1 level. Previous medical research has shown that patients [8] with high protein concentration in urine (proteinuria) could have some kind of kidney disease. However, proteinuria with minor injury, typically only albuminuria defined as detectable proteinuria in the urine between 30 and $300 \mathrm{mg} / 24 \mathrm{~h}$, can be due to vigorous physical exercise, congestive heart failure, pregnancy, alcohol abuse, or hyperthermia [9]. In addition, as it is well known that clearance of therapeutic antibodies from circulation includes nonspecific clearance through pinocytosis and proteolysis, target-mediated specific clearance, and other mechanisms such as ADAmediated clearance [10], and elimination of monoclonal antibody (mAb) through the kidney is considered insignificant as a result of the typical higher molecular weight of mAb than the glomerular filtration threshold $(55 \mathrm{kDa})$ [10]. Therefore, elimination of LY-CovMab, which has a molecular weight of approximately $147 \mathrm{kDa}$, by the kidneys is a minor pathway in its metabolism with little chance to cause the protein present in the urine. Moreover, preclinical animal studies did not find that LYCovMab causes clearly or potential injuries to the kidney either [5]. Consequently, it is speculated that the presence of protein in urine in this study was caused by normal physiological conditions of intense exercise, cold, mental stress, and other factors.

Transient blood creatinine increasing with grade 1 level was reported in three participants $(7.5 \%)$ in this study, who did not show other clinical manifestations and symptoms of kidney injury and all of them were recovered/resolved. Creatinine, as the product of muscle metabolism synthesized in the liver and stored primarily in muscle as creatine or creatine phosphate [11], is a low molecular weight substance completely filtered at the glomerulus, and undergoes minimal tubular secretion [12]. Although the blood creatinine increases sometimes suggest renal function decreases, slight increase of creatinine is normally due to diet and exercise. On the basis of the current preclinical evidence and metabolism of LY-CovMab, it is hard to infer that LY-CovMab could increase the level of blood creatinine. 
Table 4 Summary statistics of immunogenicity of LY-CovMab over time (immunogenicity set $N=40$ )

\begin{tabular}{|c|c|c|c|c|c|c|c|}
\hline Parameter & Visit & $\begin{array}{l}\text { Placebo } \\
(N=8)\end{array}$ & $\begin{array}{l}\text { LY- } \\
\text { CovMab } \\
30 \mathrm{mg} \\
(N=2)\end{array}$ & $\begin{array}{l}\text { LY- } \\
\text { CovMab } \\
150 \mathrm{mg} \\
(N=7)\end{array}$ & $\begin{array}{l}\text { LY- } \\
\text { CovMab } \\
600 \mathrm{mg} \\
(N=8)\end{array}$ & $\begin{array}{l}\text { LY- } \\
\text { CovMab } \\
1200 \mathrm{mg} \\
(N=7)\end{array}$ & $\begin{array}{l}\text { LY- } \\
\text { CovMab } \\
2400 \mathrm{mg} \\
(N=8)\end{array}$ \\
\hline \multirow{21}{*}{$\begin{array}{l}\text { Anti-LY-CovMab } \\
\text { antibodies }\end{array}$} & \multicolumn{7}{|c|}{ Day 1-Predose } \\
\hline & Negative & $8(100.0 \%)$ & $2(100.0 \%)$ & $7(100.0 \%)$ & $8(100.0 \%)$ & $6(85.7 \%)$ & $8(100.0 \%)$ \\
\hline & Positive & $0(0.0 \%)$ & $0(0.0 \%)$ & $0(0.0 \%)$ & $0(0.0 \%)$ & $1(14.3 \%)$ & $0(0.0 \%)$ \\
\hline & \multicolumn{7}{|l|}{ Day 15} \\
\hline & Negative & $8(100.0 \%)$ & $2(100.0 \%)$ & $7(100.0 \%)$ & $8(100.0 \%)$ & $7(100.0 \%)$ & $8(100.0 \%)$ \\
\hline & Positive & $0(0.0 \%)$ & $0(0.0 \%)$ & $0(0.0 \%)$ & $0(0.0 \%)$ & $0(0.0 \%)$ & $0(0.0 \%)$ \\
\hline & \multicolumn{7}{|l|}{ Day 29} \\
\hline & Negative & $8(100.0 \%)$ & $2(100.0 \%)$ & $7(100.0 \%)$ & $8(100.0 \%)$ & $6(85.7 \%)$ & $8(100.0 \%)$ \\
\hline & Positive & $0(0.0 \%)$ & $0(0.0 \%)$ & $0(0.0 \%)$ & $0(0.0 \%)$ & $0(0.0 \%)$ & $0(0.0 \%)$ \\
\hline & \multicolumn{7}{|l|}{ Day 43} \\
\hline & Negative & $8(100.0 \%)$ & $2(100.0 \%)$ & $7(100.0 \%)$ & $8(100.0 \%)$ & $5(71.4 \%)$ & $8(100.0 \%)$ \\
\hline & Positive & $0(0.0 \%)$ & $0(0.0 \%)$ & $0(0.0 \%)$ & $0(0.0 \%)$ & $0(0.0 \%)$ & $0(0.0 \%)$ \\
\hline & \multicolumn{7}{|l|}{ Day 57} \\
\hline & Negative & $8(100.0 \%)$ & $2(100.0 \%)$ & $6(85.7 \%)$ & $7(87.5 \%)$ & $6(85.7 \%)$ & $8(100.0 \%)$ \\
\hline & Positive & $0(0.0 \%)$ & $0(0.0 \%)$ & $1(14.3 \%)$ & $1(12.5 \%)$ & $0(0.0 \%)$ & $0(0.0 \%)$ \\
\hline & \multicolumn{7}{|l|}{ Day 71} \\
\hline & Negative & $8(100.0 \%)$ & $2(100.0 \%)$ & $7(100.0 \%)$ & $8(100.0 \%)$ & $5(71.4 \%)$ & $8(100.0 \%)$ \\
\hline & Positive & $0(0.0 \%)$ & $0(0.0 \%)$ & $0(0.0 \%)$ & $0(0.0 \%)$ & $1(14.3 \%)$ & $0(0.0 \%)$ \\
\hline & \multicolumn{7}{|l|}{ Day 99} \\
\hline & Negative & $8(100.0 \%)$ & $0(0.0 \%)$ & $5(71.4 \%)$ & $7(87.5 \%)$ & $4(57.1 \%)$ & $7(87.5 \%)$ \\
\hline & Positive & $0(0.0 \%)$ & $0(0.0 \%)$ & $1(14.3 \%)$ & $1(12.5 \%)$ & $1(14.3 \%)$ & $1(12.5 \%)$ \\
\hline
\end{tabular}

The denominator was the number of subjects within each group

After a single intravenous infusion of LYCovMab ranging from 150 to $2400 \mathrm{mg}$, the mean $t_{1 / 2}$ was around 28.5 days. The exposure parameters $\left(C_{\max }, \mathrm{AUC}_{0-\text { last}}\right.$, and $\left.\mathrm{AUC}_{0-\text { inf }}\right)$ increased in a linearly proportional manner as the dose increased. The PK profiles of LY-CovMab in healthy volunteers showed typical monoclonal antibody distribution and elimination characteristics. It was noticed that LYCovMab with a concentration of $1000 \mathrm{nM}$
$(150 \mu \mathrm{g} / \mathrm{mL})$ could completely neutralize viruses according to combined preclinical data of protein level blocking and pseudovirus neutralization activity in vitro. Clinically, it was found that the average blood concentration of LYCovMab at $504 \mathrm{~h}$ after a single dose of $1200 \mathrm{mg}$ injection was $169 \mu \mathrm{g} / \mathrm{mL}$, which was still higher than $1000 \mathrm{nM}(150 \mu \mathrm{g} / \mathrm{mL})$. It may suggest that one single dose of $1200 \mathrm{mg}$ LY-CovMab could 


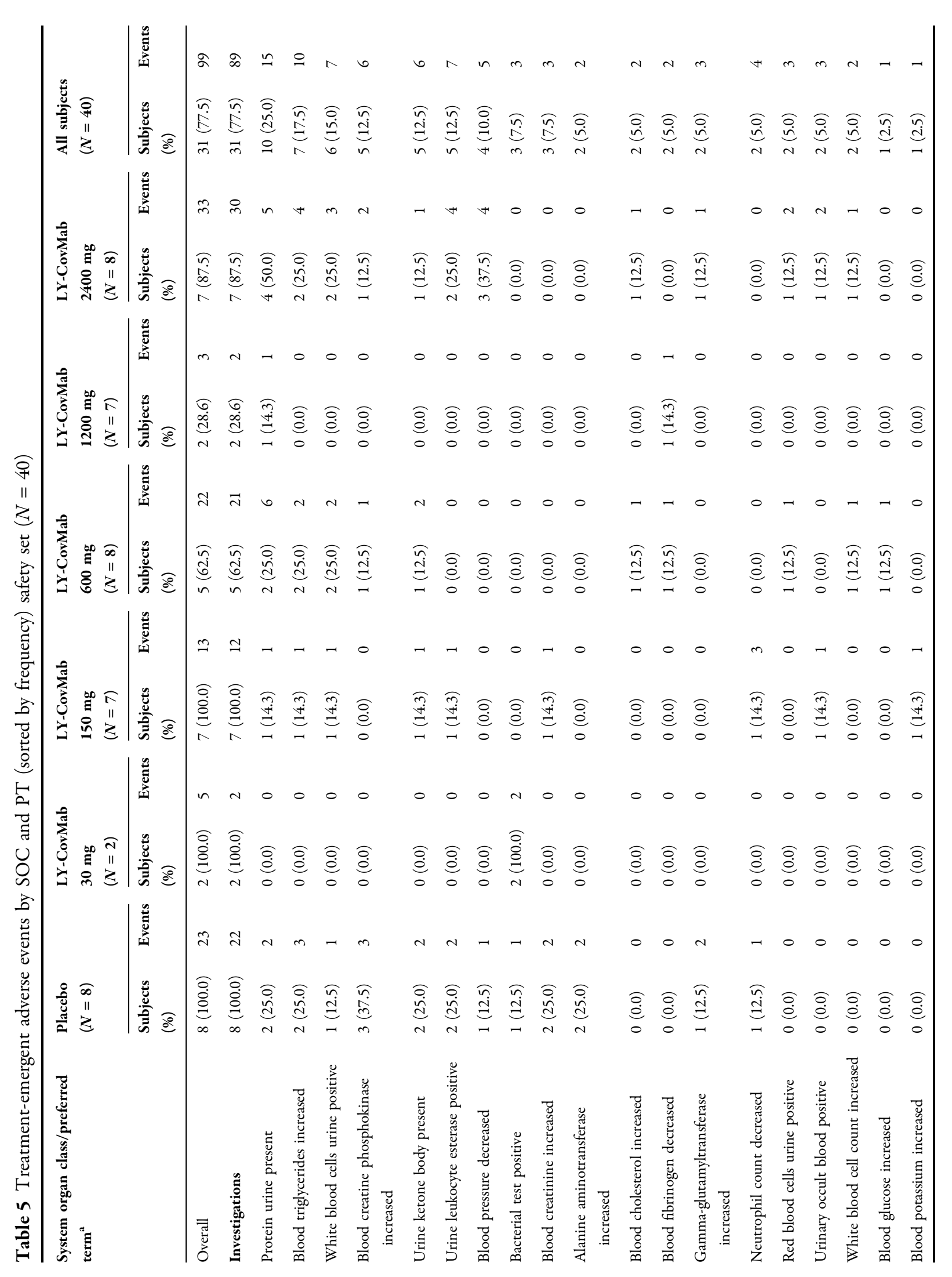




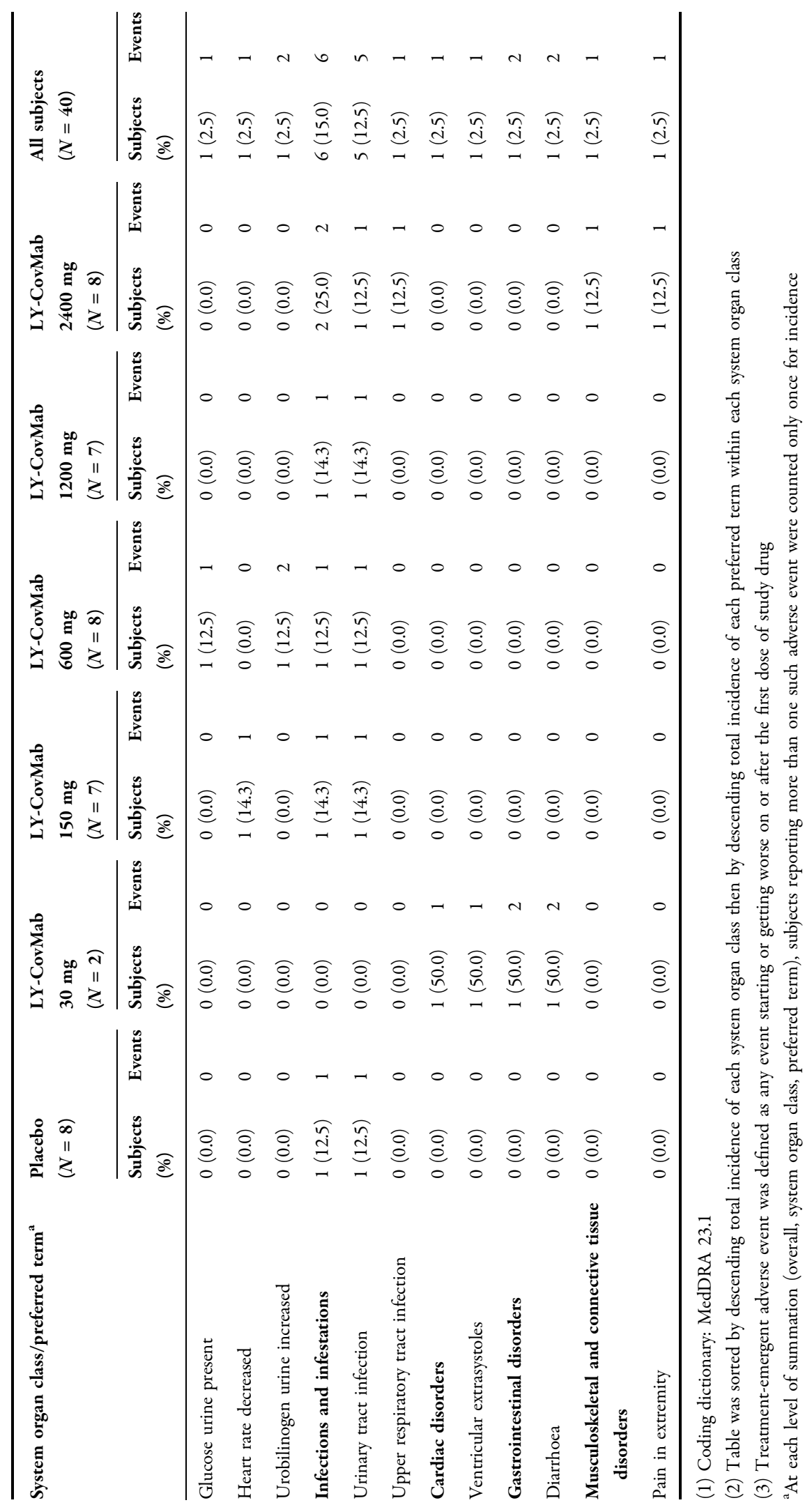




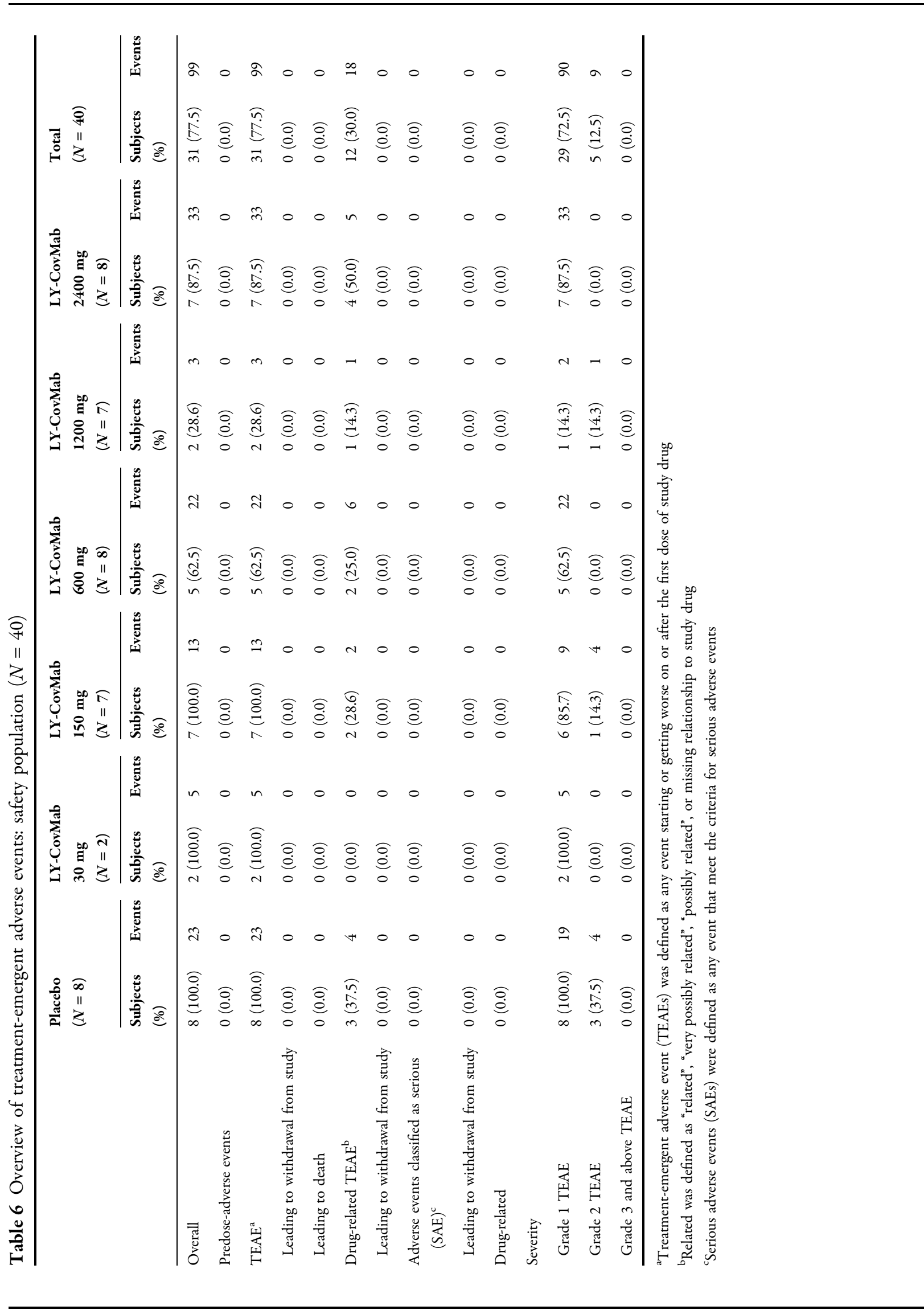




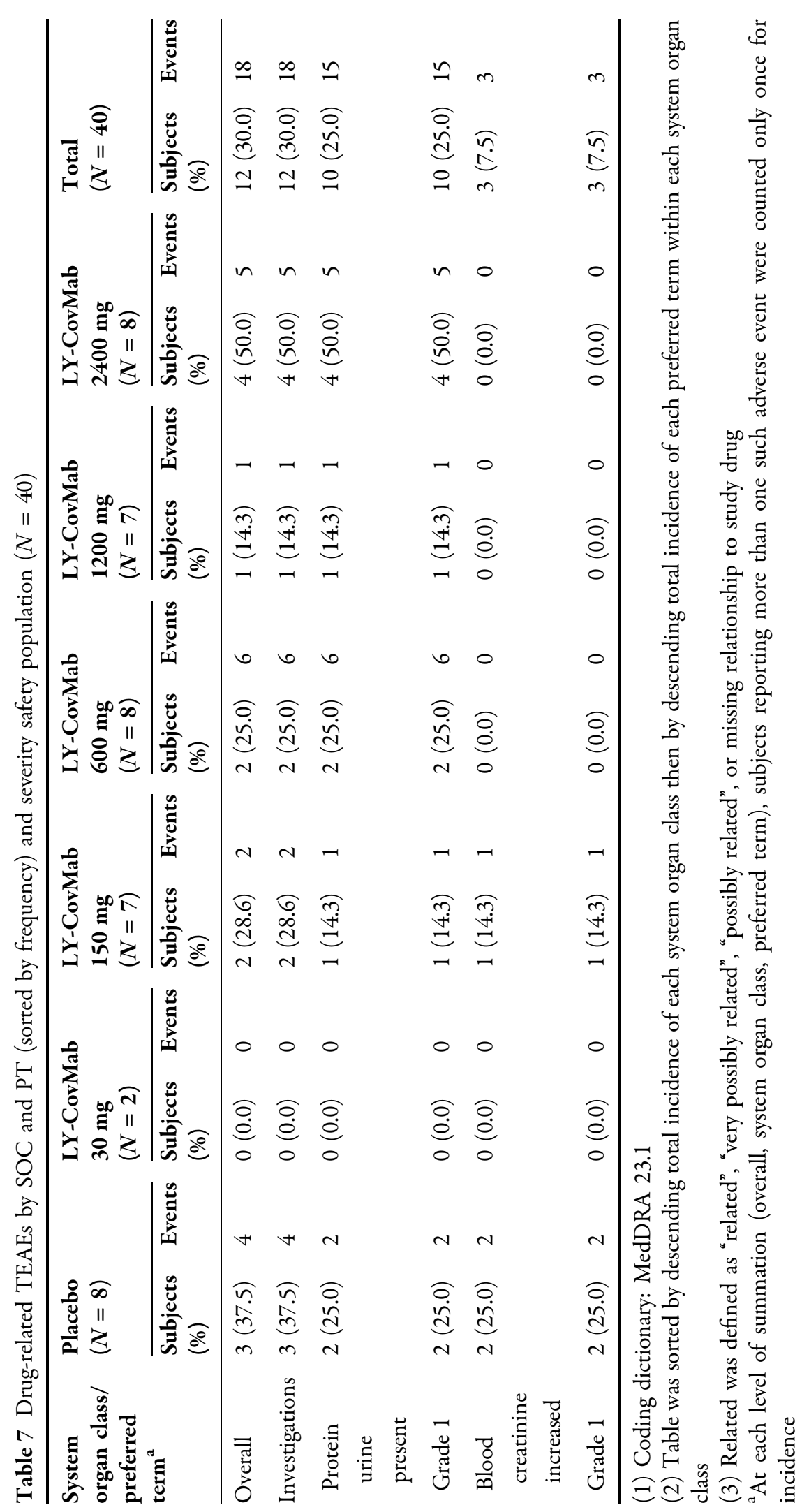


be powerful enough against SARS-CoV-2 until $540 \mathrm{~h}$ after administration.

ADA positivity was observed in five subjects who received different doses of LY-CovMab injection, with an overall ADA positive rate of $12.5 \%$. However, no impact of ADA positivity was observed on the serum concentration of LYCovMab or the safety profile. Further investigation needs to be done to understand whether positive ADA could influence LY-CovMab efficacy.

This study has limitations. First, a study with a large sample size could be more precise in demonstrating the safety and PK profile of LYCovMab. Furthermore, it would be better if some patients with mild-to-moderate COVID19 are included in the study, which may present different safety and PK characteristics of LYCovMab from those of healthy people and provide us with great horizons for further study.

\section{CONCLUSIONS}

The serum exposure of LY-CovMab ( $C_{\max }$, $\mathrm{AUC}_{0-\text { last}}, \mathrm{AUC}_{0-\text { inf }}$ ) after intravenous infusion increased in an approximately proportional manner as the dose increased from 150 to $2400 \mathrm{mg}$. The elimination half-life $\left(t_{1 / 2}\right)$ value did not differ among different dose cohorts and was estimated to be around 28.5 days. The incidence of positive ADA was $12.5 \%$. The corresponding Nab data have not been obtained. The safety results demonstrated that single doses of LY-CovMab injection at $30 \mathrm{mg}$, $150 \mathrm{mg}, 600 \mathrm{mg}, 1200 \mathrm{mg}, 2400 \mathrm{mg}$, or placebo were safe and well tolerated in Chinese healthy adults.

What is the current knowledge on the topic? LY-CovMab is a fully humanized monoclonal antibody manufactured by recombinant technology and used to counteract COVID-19. Preclinical data from LY-CovMab showed it can effectively inhibit pseudovirus and authentic virus infection in vitro and in vivo, which suggested that it could be a promising SARS-CoV-2 neutralizing antibody candidate.

\section{ACKNOWLEDGEMENTS}

We thank the participants for their involvement in this trial.

Funding. This study was supported by National Major Scientific and Technological Special Project (2020ZX09201014). Shandong Boan Biotechnology Co., Ltd., provided LYCovMab and its placebo for the clinical trial. Shandong Boan Biotechnology Co., Ltd. also funded the journal's Rapid Service Fee.

Authorship. All named authors meet the International Committee of Medical Journal Editors (ICMJE) criteria for authorship for this article, take responsibility for the integrity of the work as a whole, and have given their approval for this version to be published.

Author Contributions. Qian Zhang, Renpeng Zhou, and Jingjing Yang had full access to all the data in the study and took responsibility for the integrity of the data and the accuracy of the data analysis. Tianyi Gan, Fujia Liu, and Baihui $\mathrm{Hu}$ provided medical support during the clinical study process and were responsible for drafting and revising the manuscript. Concept and design were performed by Chao Lu and Wei $\mathrm{Hu}$.

Disclosures. Qian Zhang, Renpeng Zhou, Jingjing Yang, Changlin Dou, Tianyi Gan, Fujia Liu, Baihui Hu, Deyong Song, Chao Lu, Wei Hu all have nothing to disclose.

Compliance with Ethics Guidelines. This study was approved by the ethics committee of the Second Hospital of Anhui Medical University with the reference number SL-YW2020-113. This study was performed in accordance with the Helsinki Declaration of 1964, and its later amendments.

Data Availability. The datasets generated during and/or analyzed during the current study are available from the corresponding author on reasonable request. 
Open Access. This article is licensed under a Creative Commons Attribution-NonCommercial 4.0 International License, which permits any non-commercial use, sharing, adaptation, distribution and reproduction in any medium or format, as long as you give appropriate credit to the original author(s) and the source, provide a link to the Creative Commons licence, and indicate if changes were made. The images or other third party material in this article are included in the article's Creative Commons licence, unless indicated otherwise in a credit line to the material. If material is not included in the article's Creative Commons licence and your intended use is not permitted by statutory regulation or exceeds the permitted use, you will need to obtain permission directly from the copyright holder. To view a copy of this licence, visit http:// creativecommons.org/licenses/by-nc/4.0/.

\section{REFERENCES}

1. World Health Organization. WHO coronavirus (COVID-19) dashboard. 2021. https://covid19.who. int.

2. COVID-19 Treatment Guidelines Panel. Coronavirus disease 2019 (COVID-19) treatment guidelines. National Institutes of Health. 2021. https:// www.covid19treatmentguidelines.nih.gov/.

3. Guideline Development Group. Therapeutics and COVID-19: living guideline. Geneva: World Health Organization.

4. FDA. Emergency Use Authorization (EUAs). 2021. https://www.fda.gov/emergency-preparedness-andresponse/mcm-legal-regulatory-and-policy-frame work/emergency-use-authorization\#coviddrugs.
5. Song D, Wang W, Dong C, et al. Structure and function analysis of a potent human neutralizing antibody CA521FALA against SARS-CoV-2. Commun Biol. 2021;4:500.

6. Gottlieb RL, Nirula A, Chen P, et al. Effect of bamlanivimab as monotherapy or in combination with etesevimab on viral load in patients with mild to moderate COVID-19: a randomized clinical trial. JAMA. 2021;325:632-44.

7. Weinreich DM, Sivapalasingam S, Norton T, et al. REGN-COV2, a neutralizing antibody cocktail, in outpatients with COVID-19. N Engl J Med. 2021;384:238-51.

8. Aitekenov S, Gaipov A, Bukasov R. Review: detection and quantification of proteins in human urine. Talanta. 2020;223(Pt 1): 121718.

9. Dasgupta A, Wahed A. Clinical chemistry, immunology and laboratory quality control. Oxford: Elsevier; 2014. p. 461-83.

10. Meric O, Lin K. Tutorial on monoclonal antibody pharmacokinetics, and its considerations in early development. Clin Transl Sci. 2018;11(6):540-52.

11. Macaulay O, Nneoma A. Diabetic nephropathy and CKD-analysis of individual patient serum creatinine trajectories: a forgotten diagnostic methodology for diabetic CKD prognostication and prediction. J Clin Med. 2015;4:1348-68.

12. Ducharme MP, Smythe M, Strohs G. Drug-induced alterations in serum creatinine concentrations. Ann Pharmacother. 1993;27:622-33.

\section{Publisher's Note}

Springer Nature remains neutral with regard to jurisdictional claims in published maps and institutional affiliations 